\title{
Mixed Amiodarone-Induced Thyrotoxicosis Refractory to Medical Therapy and Plasmapheresis
}

\author{
Anupam Kotwal ${ }^{\mathrm{a}, \mathrm{d}}$, Basel Touchan ${ }^{\mathrm{a}}$, Kavita Y. Seetharaman ${ }^{\mathrm{b}}$, Richard Allen Haas ${ }^{\mathrm{b}}$, Marie Lithgow ${ }^{\mathrm{c}}$, \\ Samir Malkani ${ }^{\mathrm{b}}$
}

\begin{abstract}
Amiodarone-induced thyrotoxicosis (AIT) is associated with increased mortality in older individuals with impaired ventricular function, underlying the importance of rapid restoration and maintenance of euthyroidism. Plasmapheresis for treating thyrotoxicosis has shown mixed results. A 61-year-old gentleman was hospitalized for progressively worsening dyspnea, palpitations, tremor and generalized weakness. He had a history of atrial fibrillation, coronary artery disease, heart failure and type 2 diabetes. He had been diagnosed with AIT 4 months prior to admission, after which amiodarone was discontinued, and he was started on prednisone and methimazole which was later changed to propylthiouracil. Physical examination revealed irregular tachycardia, lid lag, fine hand tremor, proximal muscle weakness, pedal edema and a small, smooth, non-tender thyroid gland without bruit. Hormonal investigations revealed low TSH of $0.02 \mu \mathrm{IU} / \mathrm{mL}$ (reference: 0.28 - 3.89), high free T4 of $4.88 \mathrm{ng} / \mathrm{dL}$ (reference: 0.58 - 1.64), high free $\mathrm{T} 3$ of $5.4 \mathrm{pg} / \mathrm{mL}$ (reference: 2.5 - 3.9) and normal total T3 of $114 \mathrm{ng} / \mathrm{dL}$ (reference: 87 - 178). Thyroglobulin antibody, thyroid peroxidase antibody and thyroid stimulating immunoglobulin were undetectable. Echocardiogram revealed reduced left ventricle ejection fraction of $25 \%$. Ultrasound showed a bilaterally heterogeneous, hypovascular and hypoechoic thyroid. I-123 scan revealed very low uptake. These findings were suggestive of destructive type 2 AIT. However, the lack of a good response to steroids argued for type 1 AIT. Given his poor response to medical therapy and increased cardiovascular risk for an urgent thyroidectomy, plasmapheresis was instituted in an attempt to lower the thyroid hormone levels. Eight daily cycles provided only modest clinical and biochemical improvement. Subsequently, he underwent total thyroidectomy lead-
\end{abstract}

Manuscript accepted for publication April 28, 2015

${ }^{\text {aDepartment }}$ of Medicine, University of Massachusetts Medical School, 55 Lake Avenue North, Room H6-531, Worcester, MA 01655, USA

${ }^{b}$ Division of Endocrinology, Diabetes and Metabolism, Department of Medicine, University of Massachusetts Medical School, 55 Lake Avenue North, Worcester, MA 01655, USA

'Department of Pathology, University of Massachusetts Medical School, 55 Lake Avenue North, Worcester, MA 01655, USA

${ }^{\mathrm{d}}$ Corresponding Author: Anupam Kotwal, Department of Medicine, University of Massachusetts Medical School, 55 Lake Avenue North, Room H6-531, Worcester, MA 01655, USA. Email: anupam.kotwal@umassmemorial.org

doi: http://dx.doi.org/10.14740/jem278w ing to improvement of thyrotoxicosis. Histopathology of the thyroid demonstrated intrafollicular histiocytes, patchy fibrosis and involuted follicles, suggestive of destructive thyroiditis. On follow-up 2 months later, he remained clinically euthyroid on levothyroxine replacement with stable cardiac status. This case represents mixed/indeterminate AIT of protracted duration refractory to thionamides and steroids. Plasmapheresis did not provide clinical improvement and only modestly lowered the free T4 level. Euthyroidism was finally achieved following total thyroidectomy. This finding suggests the limitations of utilizing plasmapheresis as a therapeutic modality prior to definitive treatment in AIT.

Keywords: Amiodarone; Thyrotoxicosis; Plasmapheresis; Thyroidectomy; Euthyroidism

\section{Introduction}

Amiodarone is a widely used class three anti-arrhythmic agent that contains $37 \%$ iodine by weight. Thyroid dysfunction secondary to amiodarone can manifest as hypothyroidism or thyrotoxicosis, and relates to the high iodine content within the molecule as well as to several unique intrinsic properties [1]. Amiodarone-induced thyrotoxicosis (AIT) has been associated with increased mortality in older individuals with impaired ventricular function [1], underlying the importance of rapid restoration and maintenance of euthyroidism in this condition. Amiodarone has been reported to cause thyroid dysfunction in $15-20 \%$ of cases [1-3], with the incidence of thyrotoxicosis being approximately $5 \%[3,4]$. Plasmapheresis as a treatment option for thyrotoxicosis refractory to medical therapy has shown mixed results.

\section{Case Report}

A 61-year-old Caucasian gentleman was hospitalized for progressively worsening dyspnea, palpitations, tremor and generalized weakness of 2 months duration. He had a history of atrial fibrillation, coronary artery disease, ischemic cardiomyopathy, refractory heart failure and type 2 diabetes mellitus. He had been diagnosed with thyrotoxicosis 4 months prior to admission, which was attributed to long-standing amiodarone 
Table 1. The Trend of Thyroid Profile During the Hospital Stay

\begin{tabular}{llllll}
\hline Hormonal investigations & On presentation & $\begin{array}{l}\text { After } \mathbf{8} \text { cycles of } \\
\text { plasmapheresis }\end{array}$ & $\mathbf{2 4}$ h post-surgery & 72 h post-surgery & Reference range \\
\hline TSH $(\mu \mathrm{IU} / \mathrm{mL})$ & 0.02 & 0.04 & 0.08 & Not tested & $0.28-3.89$ \\
\hline Free T4 $(\mathrm{ng} / \mathrm{dL})$ & 4.88 & 4.54 & 3.25 & 1.72 & $0.58-1.64$ \\
Free T3 $(\mathrm{pg} / \mathrm{mL})$ & 5.4 & 5.3 & 3.7 & 61 & $2.5-3.9$ \\
Total T3 $(\mathrm{ng} / \mathrm{dL})$ & 114 & 111 & 67 & 2.1 & $87-178$ \\
\hline
\end{tabular}

therapy for atrial fibrillation. Following this diagnosis, amiodarone had been discontinued, and prednisone and methimazole were initiated. Daily doses as high as $40 \mathrm{mg}$ of methimazole and $60 \mathrm{mg}$ of prednisone were not effective at controlling the thyrotoxicosis. Hence, the methimazole was later changed to propylthiouracil (PTU); however, even a dose as high as 200 $\mathrm{mg}$ three times a day offered only minimal improvement, and the patient continued to remain symptomatic. Therapy with lithium had also been attempted but discontinued after 2 weeks due to worsening renal function.

On presentation to the hospital, his physical examination was significant for irregular tachycardia, bilateral lid lag, fine hand tremor, proximal muscle weakness, patellar hyperreflexia and bilateral pitting pedal edema. The thyroid gland was noted to be small, smooth and non-tender without bruit or nodules. Hormonal investigations revealed a low TSH of 0.02 $\mu \mathrm{IU} / \mathrm{mL}$ (reference range is $0.28-3.89$ ), elevated free T4 of $4.88 \mathrm{ng} / \mathrm{dL}$ (reference range is $0.58-1.64$ ), elevated free T3 of $5.4 \mathrm{pg} / \mathrm{mL}$ (reference range is $2.5-3.9$ ) and normal total $\mathrm{T} 3$ of $114 \mathrm{ng} / \mathrm{dL}$ (reference range is 87 - 178). This thyroid profile was consistent with thyrotoxicosis. Thyroglobulin antibody (TG Ab), thyroid peroxidase antibody (TPO Ab) and thyroid stimulating immunoglobulin (TSI) were undetectable, making autoimmune etiologies unlikely. A transthoracic echocardiogram revealed a reduced left ventricle ejection fraction of $25 \%$. Thyroid ultrasound was then performed which demonstrated a bilaterally heterogeneous, hypovascular and hypoechoic gland. Thyroid scan with radioactive iodine-123 revealed a very low uptake of $1.5 \%$ at $6 \mathrm{~h}$ and $0.9 \%$ at $24 \mathrm{~h}$ (expected is $8-20 \%$ at $6 \mathrm{~h}$ and $10-35 \%$ at $24 \mathrm{~h}$ ). These findings were suggestive of destructive type 2 AIT. However, the lack

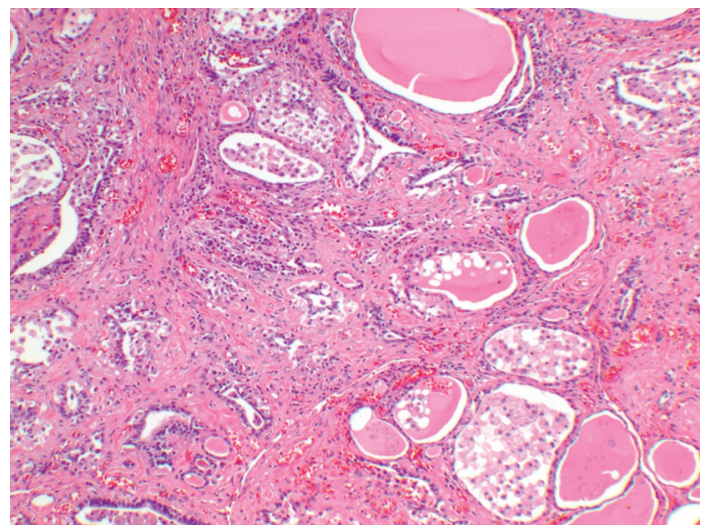

Figure 1. Thyroid gland histopathology demonstrates intra-follicular histiocytes, patchy fibrosis and involuted follicles. of a good response to steroid therapy argued for type 1 AIT. Medical therapy with PTU, prednisone and propranolol was continued. Given his poor response to medical therapy and increased cardiovascular risk for an urgent thyroidectomy, plasmapheresis was instituted in an attempt to lower the thyroid hormone levels prior to thyroidectomy. Eight daily cycles of plasmapheresis, with increasing volumes of plasma exchange provided only modest clinical and biochemical improvement with free T4 eventually decreasing only modestly to $4.54 \mathrm{ng} /$ $\mathrm{dL}$ (reference range is $0.58-1.64$ ). The patient continued to remain symptomatic. Subsequently, he underwent a total thyroidectomy, during which a hard thyroid on both sides with severe fibrosis and adhesions especially over the trachea was noted and removed. The right lobe measured $5.5 \times 2.5 \times 2.5$ $\mathrm{cm}$ and the left lobe measured $4 \times 3 \times 1.5 \mathrm{~cm}$. Within $24 \mathrm{~h}$, his free T4 level decreased to $3.25 \mathrm{ng} / \mathrm{dL}$, free $\mathrm{T} 3$ decreased to $3.7 \mathrm{pg} / \mathrm{mL}$ and total T3 decreased to $67 \mathrm{ng} / \mathrm{dL}$ (Table 1). Histopathology of the thyroid gland demonstrated intra-follicular histiocytes, patchy fibrosis and involuted follicles, suggestive of destructive thyroiditis (Fig. 1). On follow-up 2 months later, he remained clinically euthyroid on levothyroxine replacement and maintained a stable cardiac status.

\section{Discussion}

The diagnosis of AIT is based on the presence of increased free thyroid hormone concentrations and suppressed TSH levels in the setting of amiodarone therapy. There are two main forms of AIT: type 1, a form of iodine-induced hyperthyroidism due to excess hormone synthesis, and type 2, a drug-induced destructive thyroiditis causing excess hormone release [1]. However, mixed/indefinite forms exist that may be caused by both the pathogenic mechanisms. Type 1 AIT usually occurs in abnormal thyroid glands, whereas type 2 AIT develops in apparently normal thyroid glands or small goiters. Thyroid radioactive iodine-123 uptake values are usually very low in type 2 AIT, whereas uptake values are most commonly low or low-normal, but sometimes normal or increased despite the iodine load in type 1 AIT. Ultrasound usually demonstrates absent or decreased vascularity in type 2 and hyper-vascularity in type 1 AIT. Mixed/indefinite forms may have features of both AIT types (Table 2). In our patient, the hypovascular thyroid on ultrasound and very low uptake on RAIU scan were features of type 2 AIT; however, the lack of a good response to steroids favored type 1 AIT. Histopathology demonstrated features consistent with destructive thyroiditis (Fig. 1). Hence, this was most likely a case of mixed/indeterminate AIT. 
Table 2. Characteristics of the Different Types of Amiodarone-Induced Thyrotoxicosis (AIT)

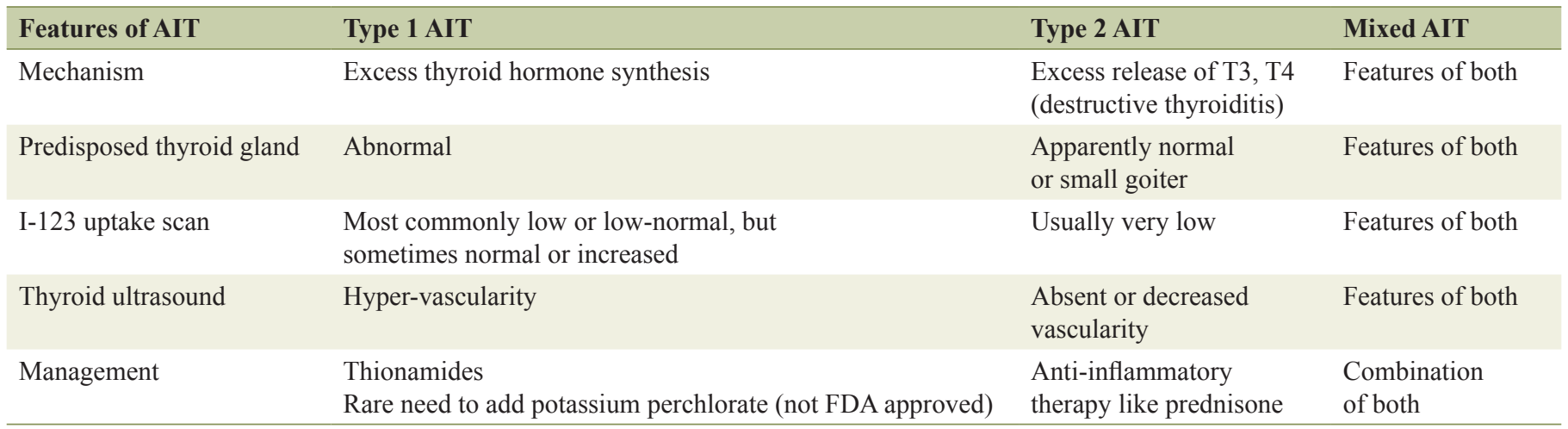

Management guidelines of the American Thyroid Association (ATA) and American Association of Clinical Endocrinologists (AACE) recommend methimazole for treatment of type 1 AIT (with a rare need to add potassium perchlorate which is not FDA approved for this indication), whereas type 2 is better treated with anti-inflammatory therapy such as prednisone $[5,6]$. The most difficult challenge is represented by mixed/ indefinite forms of AIT. In these cases, both the pathogenic mechanisms (increased thyroid hormone synthesis and thyroid hormone discharge due to glandular damage) are likely operating. Thus, the best treatment is represented by a combination of thionamides (with or without potassium perchlorate) and oral glucocorticoids [1]. Radioactive iodine-123 as a treatment modality is usually not feasible in AIT due to low uptake values [1].

The need for amiodarone discontinuation is controversial; the decision to stop amiodarone in the setting of thyrotoxicosis should be determined on an individual basis in consultation with a cardiologist, based on the presence or absence of effective alternative anti-arrhythmic therapy [5]. The effects of amiodarone on the thyroid may last for several months after amiodarone discontinuation due to its long half-life [1]; accordingly, drug withdrawal might not influence the response to medical therapy in the short term [1]. The amiodarone in our patient had been discontinued after the development of thyrotoxicosis. The use of lithium has been proposed for AIT [7], but the evidence to support its effectiveness is limited. The ATA and AACE guidelines do not recommend the use of plasmapheresis, most likely because of limited data to support its therapeutic effectiveness. It has proven useful in some cases $[8,9]$ but has failed in others [10]. Our patient's thyrotoxicosis did not improve even with high doses of PTU, prednisone and propranolol. Given his poor response to medical therapy and increased cardiovascular risk for an urgent thyroidectomy, plasmapheresis was instituted in an attempt to reduce the thyroid hormone levels prior to thyroidectomy. Eight daily cycles of plasmapheresis, with increasing volumes of plasma exchange provided only modest clinical and biochemical improvement. Thyroidectomy has shown to be a valid option in cases resistant to medical therapy or when a rapid restoration of euthyroidism is advisable $[1,5]$. In our case as well, euthyroidism was finally achieved after the patient underwent a total thyroidectomy.

\section{Conclusions}

Thyrotoxicosis due to amiodarone therapy is usually differentiated into type 1 or type 2 but some cases are classified as mixed/indeterminate as they demonstrate features of both types of AIT. Mixed types of AIT can be challenging both to diagnose and treat, often requiring a combination of thionamides, steroids and beta-blocking agents. AIT may present as a severe condition with protracted duration and remain refractory to medical therapy. Plasmapheresis has its limitations as a therapeutic modality for the thyrotoxic state. Thyroidectomy remains the definitive management of AIT and should be instituted sooner rather than later in a patient suffering from this condition.

\section{Conflict of Interest}

The authors have no conflicts of interest.

\section{References}

1. Bogazzi F, Bartalena L, Martino E. Approach to the patient with amiodarone-induced thyrotoxicosis. J Clin Endocrinol Metab. 2010;95(6):2529-2535.

2. Batcher EL, Tang XC, Singh BN, Singh SN, Reda DJ, Hershman JM. Thyroid function abnormalities during amiodarone therapy for persistent atrial fibrillation. Am J Med. 2007;120(10):880-885.

3. Farhan H, Albulushi A, Taqi A, Al-Hashim A, Al-Saidi K, Al-Rasadi K, Al-Mazroui A, et al. Incidence and pattern of thyroid dysfunction in patients on chronic amiodarone therapy: experience at a tertiary care centre in oman. Open Cardiovasc Med J. 2013;7:122-126.

4. Uchida T, Kasai T, Takagi A, Sekita G, Komiya K, Takeno K, Shigihara N, et al. Prevalence of amiodarone-induced thyrotoxicosis and associated risk factors in Japanese patients. Int J Endocrinol. 2014;2014:534904.

5. Bahn RS, Burch HB, Cooper DS, Garber JR, Greenlee MC, Klein I, Laurberg P, et al. Hyperthyroidism and other causes of thyrotoxicosis: management guidelines 
of the American Thyroid Association and American Association of Clinical Endocrinologists. Endocr Pract. 2011;17(3):456-520.

6. Bogazzi F, Tomisti L, Rossi G, Dell'Unto E, Pepe P, Bartalena L, Martino E. Glucocorticoids are preferable to thionamides as first-line treatment for amiodaroneinduced thyrotoxicosis due to destructive thyroiditis: a matched retrospective cohort study. J Clin Endocrinol Metab. 2009;94(10):3757-3762.

7. Dickstein G, Shechner C, Adawi F, Kaplan J, Baron E, Ish-Shalom S. Lithium treatment in amiodarone-induced thyrotoxicosis. Am J Med. 1997;102(5):454-458.

8. Diamond TH, Rajagopal R, Ganda K, Manoharan A, Luk A. Plasmapheresis as a potential treatment option for amiodarone-induced thyrotoxicosis. Intern Med J. 2004;34(6):369-370; author reply 370-361.

9. Ligtenberg J, Tulleken J, Zijlstra J. Plasmapheresis in thyrotoxicosis. Ann Intern Med. 1999;131(1):71-72.

10. Samaras K, Marel GM. Failure of plasmapheresis, corticosteroids and thionamides to ameliorate a case of protracted amiodarone-induced thyroiditis. Clin Endocrinol (Oxf). 1996;45(3):365-368. 